\title{
The Wind Load Prediction of Asymmetric Semi-submersible Lifting Platform based on CFD
}

\author{
Zhenqiu Yao, Xiangyu Tang, Hongjie Ling
}

\begin{abstract}
In order to accurately predict the wind load of asymmetric marine structures, the $3 \mathrm{~d}$ model of an asymmetric semi-submersible lifting platform was established by using SolidWorks software in this paper.The wind load based on 1:25 scale model was numerically predicted by using CFD software FINE/MARINE .The numerical simulation wind load is converted into the actual value through the similarity theory, and compared with the calculated value of CCS specification. The results show that The change trend of longitudinal force and transverse force of wind direction Angle of $0^{\circ} \sim \mathbf{1 8 0}^{\circ}$ and $180^{\circ} \sim 360^{\circ}$ basically remains the same, but the values are not the same due to the asymmetry of the platform. In addition, the standard calculation method cannot fully consider the effect of shading effect among the superstructure components, so the result is larger than the numerical simulation value. When the fluid meets the vertical windward surface of the superstructure, its velocity will plummet and flow into all directions. This paper provides an effective method for wind load prediction of asymmetric Marine structures and has certain reference value for the design and research of asymmetric semi-submersible lifting platform.
\end{abstract}

Index Terms - asymmetric semi-submersible lifting platform, wind load, CFD, shading effect.

\section{INTRODUCTION}

Semi-submersible lifting platform has been widely used in the aspects of offshore hoisting, submarine pipeline laying, and offshore platform removal, etc., by virtue of its large lifting capacity, strong anti-storm ability, wide range of working water depth and other advantages [1-2].In the design of offshore platform, wind load is one of the important loads to be considered, which has a significant impact on the offshore structure of the platform. At present, the research on wind load in the world mainly consists of 4 methods: field measurement, wind tunnel test, numerical simulation, and standard calculation. Due to its high reliability, wind tunnel test is often used as an important means of wind load research [3], but the test takes a long time. Therefore, the research of ocean typhoon load is still based on standard calculation and numerical simulation.

Furnes calculated the wind loads of offshore oil drilling platforms at different wind angles and obtained the results that are very consistent with the wind tunnel test data [4].Ma zhe applied FLUENT software to conduct numerical simulation calculation of wind load of semi-submersible platform with working water depth of $3000 \mathrm{~m}$, and calculated wind load results under two conditions: solid derrick and hollow derrick respectively [5]. Lin yi took the 400-foot jack-up drilling platform independently developed by CIMC as an example, measured its wind load in the field through wind tunnel experiment, and conducted numerical simulation calculation of its load based on CFD method. The results show that the numerical simulation results are smaller than the standard results and similar to the wind tunnel experiments, which can provide support for the preliminary design of the platform [6].Tang jing compared the calculation methods among three classification societies, ABS, DNV and CCS for a jackup platform load, and compared the results of wind load calculation [7].

In this paper, on the basis of existing studies, the wind load of an asymmetric semi-submersible lifting platform was numerically simulated and calculated based on FINE/MARINE software. The load size of the platform under different wind directions and the flow field distribution near the platform were emphatically studied, and the calculated results were compared and analyzed with CCS specifications.

\section{THE ESTABLISHMENT AND MESHING OF MODEL AND COMPUTATION DOMAIN}

\section{A. Model}

For an asymmetric semi-submersible lifting platform as the research object, its main parameters are shown in Table 1. The information according to the parameter information, using the geometrical model of solidworks software to complete platform, to improve the efficiency of calculation, this article only to establish a platform and the platform non-immersed areas less affected to the wind load part of component to simplify or delete, complete for wind load calculation model is shown in Figure 1.

Table 1. Main parameters of asymmetric semisubmersible lifting platform

\begin{tabular}{ccc}
\hline The parameter name & The numerical & unit \\
\hline Principal dimensions & $137.75 \times 81 \times 42.8$ & $\mathrm{~m}$ \\
The floating body & $137.75 \times 19.5 \times 12$ & $\mathrm{~m}$ \\
Leg floating body & $122 \times 13.5 \times 12$ & $\mathrm{~m}$ \\
The main pillars & $22.5 \times 19.5 \times 18$ & $\mathrm{~m}$ \\
Leg column & $16.5 \times 13.5 \times 18$ & $\mathrm{~m}$ \\
The upper hull & $81 \times 81 \times 12.8$ & $\mathrm{~m}$ \\
\hline
\end{tabular}

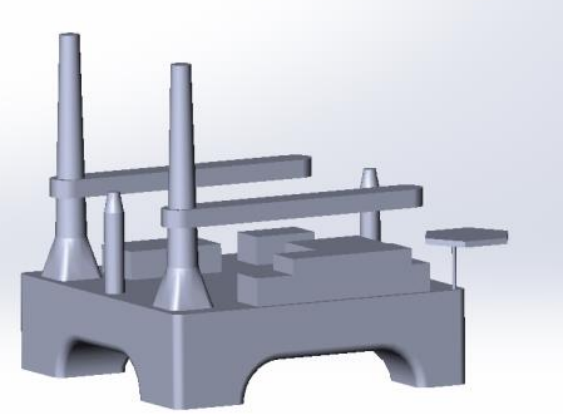

Figure $13 \mathrm{D}$ model of the aquatic part of asymmetric semisubmersible lifting platform 


\section{B. Computational Domain And Grid Division}

The solidworks model is saved as $\mathrm{x} \_\mathrm{t}$ format and imported into Hexpress software to establish the calculation domain. In order to minimize the impact of the boundary on the calculation results, the calculation domain is set as a cuboid with a large enough boundary, 3 times the length from the bow, 6 times the length from the stern, and 2 times the length from both sides of the ship's side. The model is placed at the bottom of the calculation domain, and the bottom is 3 times the length from the upper boundary. In order to accurately and conveniently simulate the wind load of the platform under different wind directions, a cylindrical domain was built around the platform, which was defined as the calculation inner domain, and the outer domain was defined as the Boolean minus calculation inner domain. The load at different wind direction angles can be calculated by rotating the inner domain. Under different wind directions, the number of grids varies but not significantly, with a total of 1.2 million grids. Figure 2 shows the computing domain, and Figure 3 show the grid division of platform surface.

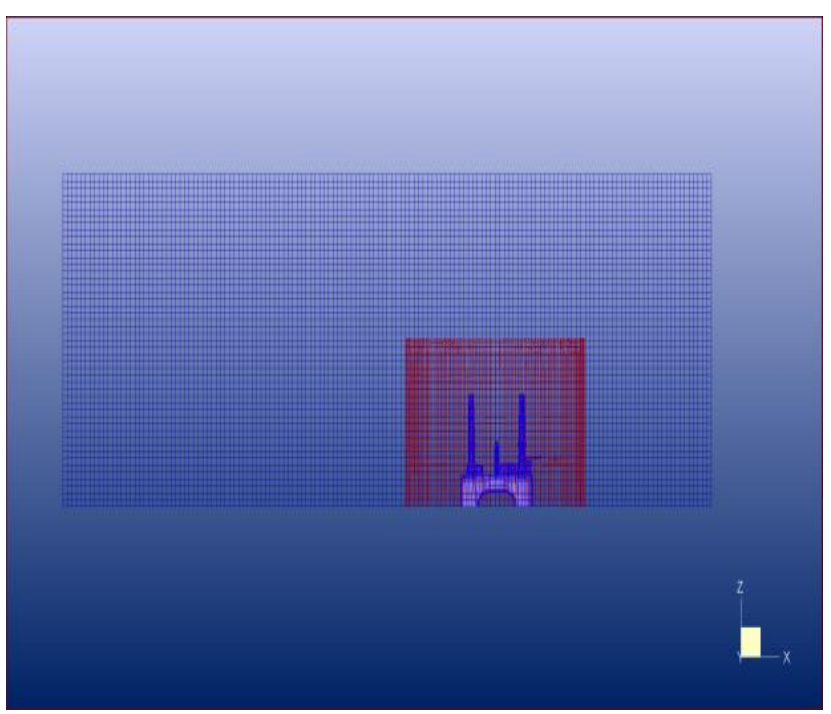

Figure 2 The Computing Domain

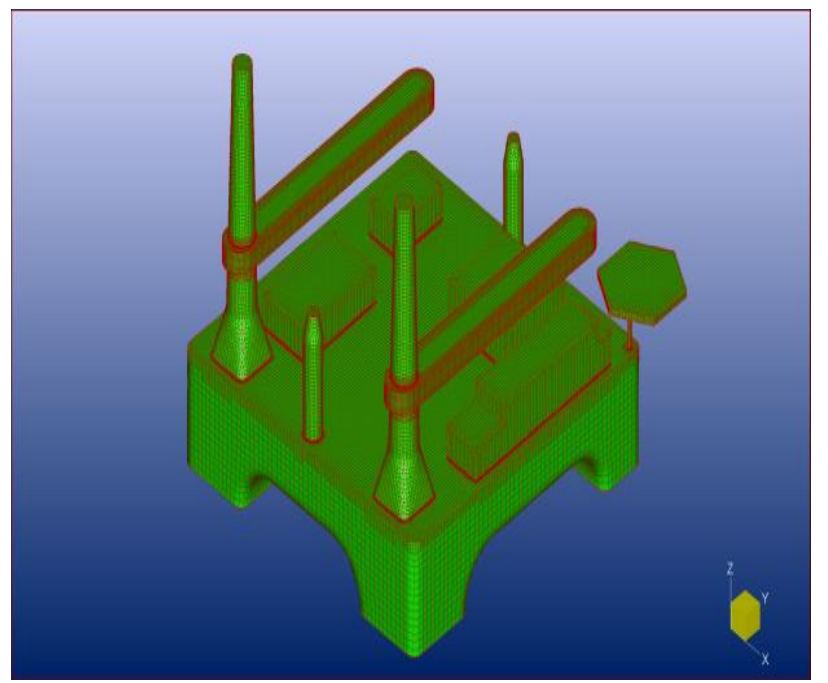

Figure 3 The Grid Division of Platform Surface.

III. NUMERICAL SIMULATION

\section{A. The Governing Equation}

Local structures of Marine platforms are mostly blunt bodies, and the numerical simulation type of the surrounding flow field is similar to the real air flow. The navier-stocks (RANS) equation for the entire flow field is adopted in Reynolds equation:

$\frac{\partial \rho}{\partial t}+\frac{\partial}{\partial z_{i}}\left(\rho u_{i}\right)=0$

$\frac{\partial}{\partial t}\left(\rho u_{i}\right)+\frac{\partial}{\partial x_{j}}\left(\rho u_{i} u_{j}\right)=-\frac{\partial p}{\partial x_{i}}+\frac{\partial}{\partial x_{j}}\left(\mu \frac{\partial u_{i}}{\partial z_{j}}-\rho \overline{u_{i}^{\prime}} \overline{u_{j}^{\prime}}\right)+S_{i}$

In the formula , $i, j=1,2,3$; air density $\mathrm{p}=1.218 \mathrm{Kg} / \mathrm{m}^{3}$; dynamic viscosity $\mu=1.785 \times 10^{-5} \mathrm{~Pa} \times \mathrm{s}$ 。

\section{B. Calculate Working Condition And Numerical Calculation Method}

The origin of the model coordinate system 0 is located at the intersection of the mid-longitudinal section, mid-transverse section and waterplane of the platform, with the $\mathrm{X}$-axis parallel waterplane pointing to the bow and $\mathrm{Y}$-axis to the left chord. The wind direction Angle was defined as the included Angle between the wind direction and the $\mathrm{X}$-axis, and the draft $d=20 \mathrm{~m}$, wind speed $\mathrm{v}=20 \mathrm{~m} / \mathrm{s}$, wind Angle $0^{\circ} \sim 360^{\circ}$, and Angle interval $15^{\circ}$ were calculated for the platform under 25 operating conditions. The FINE/MARINE software based asymmetric semi-submersible crane typhoon load prediction parameters are set as follows: three-dimensional unsteady single-phase flow; K-omega (SST-Menter) turbulence model was used, and the value of $\mathrm{k}$ and omega was related to Reynolds number. Fixed six degrees of freedom of the platform, given the inlet, outlet and left and right boundary velocity of the calculation domain, the upper boundary was set as the specified pressure condition, and the lower boundary was set as the velocity far field with the default value of 0 .The turbulence equation is discretized by AVLSMART scheme. The momentum equation is discretized by AVLSMART scheme.

\section{STANDARD CALCULATION OF WIND LOAD}

The specification of CCS is one of the most important methods for wind load prediction. According to the CCS specification, the steady wind acting on the platform can be calculated as follows:

$$
F_{w}=1 / 2 \rho \sum\left(\mathrm{C}_{s i} \mathrm{C}_{h i} \mathrm{~A}_{i}\right) V_{w}^{2}
$$

In the formula: $\rho$ is the density of air; $V_{w}$ is the design wind speed; $C_{s}$ is the shape coefficient, which is determined by Table $2 ; C_{h}$ is the height coefficient, which is determined by Table $3 ;$ Is the area under wind.

Table 2 Shape coefficient

\begin{tabular}{cc}
\hline Shape & Shape factor \\
\hline Spherical & 0.40 \\
Cylindrical & 0.50 \\
Hull & 1.00 \\
Deck House & 1.00 \\
Isolated Structure & 1.50 \\
Beams & 1.30 \\
Below Deck Area & 1.00 \\
Drilling Rigs & 1.25 \\
\hline
\end{tabular}


Table3. Height coefficient

\begin{tabular}{cc}
\hline Height $(\mathrm{m})$ & Height factor \\
\hline $0-15.3$ & 1.00 \\
$15.3-30.5$ & 1.10 \\
$30.5-46.0$ & 1.20 \\
$46.0-61.0$ & 1.30 \\
$61.0-76.0$ & 1.37 \\
$76.0-91.5$ & 1.43 \\
$91.5-106.5$ & 1.48 \\
$106.5-122.0$ & 1.52 \\
$122.0-137.0$ & 1.56 \\
$137.0-152.5$ & 1.60 \\
$152.5-167.5$ & 1.63 \\
$167.5-183.0$ & 1.67 \\
$183.0-198.0$ & 1.70 \\
$198.0-213.5$ & 1.72 \\
$213.5-228.5$ & 1.75 \\
$228.5-244.0$ & 1.77 \\
$244.0-259.0$ & 1.79 \\
Above 259.0 & 1.80 \\
\hline
\end{tabular}

\section{CALCULATION Results AND ANALysis}

\section{A. Comparative Analysis Of Loads At Different Wind Directions}

According to the similarity theory, the simulated wind load is converted into the wind load of the actual size platform, and the actual typhoon load is compared with the calculated value of CCS specification. Figure 3 and Figure 4 respectively show the longitudinal force and transverse force of the platform at different wind angles. According to Figure 3 and Figure 4, some rules of the loading on the platform can be summarized: 1) longitudinal force: the calculated values of the longitudinal force of wind load in CCS specification reach the peak value when the wind direction is $15^{\circ}, 165^{\circ}, 195^{\circ}$ and $345^{\circ}$ respectively. By comparing several peaks, it can be found that the longitudinal force of the platform is the largest when the wind direction is $165^{\circ}$. The peak value of CFD calculation appears in $30^{\circ}, 135^{\circ}, 210^{\circ}$ and $330^{\circ}$. By comparison, the longitudinal force of the platform is the largest when the wind direction is $30^{\circ} .2$ ) lateral force: the calculated values of wind load longitudinal force (CCS) reach the peak value when the wind direction is $60^{\circ}, 120^{\circ}, 240^{\circ}$ and $300^{\circ}$ respectively, that is, $\pm 60^{\circ}$ when the wind direction is downwind or downwind. By comparison, the lateral force of the platform is the largest when the wind direction is $60^{\circ}$, and the CFD calculation value is not very obvious. It appears near $30^{\circ}, 150^{\circ}, 210^{\circ}$ and $315^{\circ}$, and the lateral force is the largest when the wind direction is $315^{\circ} .3$ ) the change trend of longitudinal and transverse force of wind direction Angle $0^{\circ} \sim 180^{\circ}$ and $180^{\circ} \sim 360^{\circ}$ is basically the same, but the values are not the same due to the asymmetry of the platform.

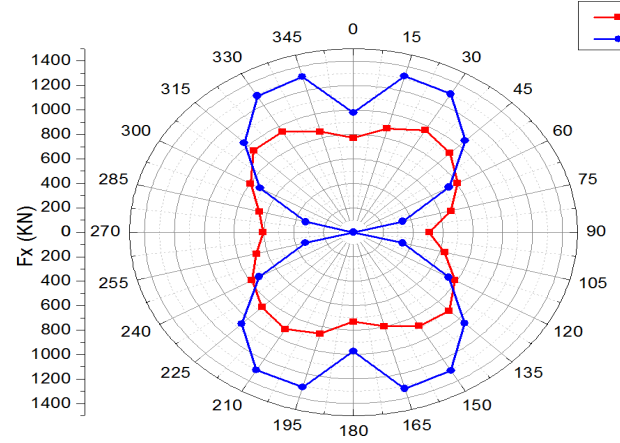

Figure 4 Longitudinal Forces at Different Wind Angles

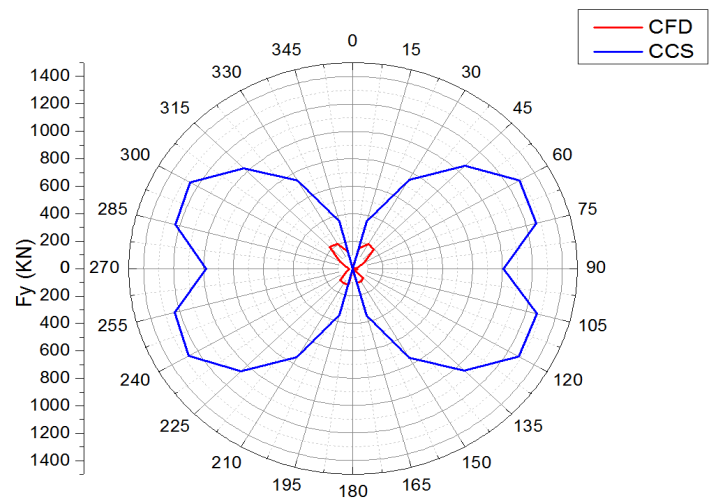

Figure 5 Transverse Forces at Different Wind Angles

Table 4 shows the comparison between the calculated results of wind load specifications and the numerical simulation results. It can be seen from the table that the calculated results of specifications are larger than the numerical simulation results.Observation standard calculation results with the results of numerical simulation, the wind direction Angle $0^{\circ} \sim 180^{\circ}$ and $180^{\circ} \sim 360^{\circ}$ error of both types of showed a trend of decrease after the first increase, and the wind load force CCS specification values calculated respectively in the direction of the wind $30^{\circ}, 150^{\circ}$, $210^{\circ}$ and $330^{\circ}$ when peak, and $150^{\circ}$ direction when the resultant force is the largest, the results of numerical simulation is slightly different, respectively, in the direction of the wind $30^{\circ}, 135^{\circ}, 210^{\circ}$ and $330^{\circ}$ when peak, and $30^{\circ}$ of the wind force is the largest. In addition, when the wind is normal, the standard results are $26.8 \%$ larger than the numerical simulation results, and when the wind is transverse, the standard results are $98.4 \%$ larger than the numerical simulation results. On the one hand, it indicates that the mutual shading of the superstructure is the most obvious under the windward and downwind conditions of the platform On the other hand, it also reflects that the normative calculation method cannot fully consider the influence of the shading effect between the components of the superstructure before and after.

Table 4. Comparison of calculated results of wind load specification and numerical simulation results

\begin{tabular}{cccccccc}
\hline $\begin{array}{c}\text { Angle } \\
/\left(^{8}\right)\end{array}$ & $\begin{array}{c}\text { Resultant } \\
\text { force/kN }\end{array}$ & $\begin{array}{c}\text { Differ } \\
\text { ences } \\
/ \%\end{array}$ & $\begin{array}{c}\text { Angle } \\
/\left({ }^{8}\right)\end{array}$ & \multicolumn{2}{c}{$\begin{array}{c}\text { Resultant } \\
\text { force } / \mathrm{kN}\end{array}$} & $\begin{array}{c}\text { Differ } \\
\text { ences } \\
/ \%\end{array}$ \\
\hline 0 & CCS & CFD & & & CCS & CFD & \\
\hline 15 & 1368 & 890 & 53.7 & 210 & 1500 & 922 & 62.7 \\
30 & 1503 & 986 & 52.4 & 225 & 1498 & 874 & 71.4 \\
45 & 1496 & 935 & 60.0 & 240 & 1464 & 786 & 86.3 \\
60 & 1483 & 811 & 82.9 & 255 & 1280 & 674 & 89.9 \\
75 & 1316 & 676 & 94.7 & 270 & 981 & 605 & 62.1 \\
90 & 1006 & 507 & 98.4 & 285 & 1274 & 655 & 94.5 \\
105 & 1320 & 633 & 108.5 & 300 & 1450 & 802 & 80.8 \\
120 & 1476 & 783 & 88.5 & 315 & 1464 & 970 & 50.9 \\
135 & 1488 & 912 & 63.2 & 330 & 1485 & 971 & 52.9 \\
150 & 1506 & 890 & 69.2 & 345 & 1364 & 862 & 58.2 \\
165 & 1371 & 803 & 70.7 & 360 & 976 & 770 & 26.8 \\
180 & 973 & 729 & 33.5 & & & & \\
\hline
\end{tabular}




\section{B. Detailed Analysis Of Flow Field}

Taking the $0^{\circ}$ wind Angle as an example, the flow field distribution near the platform surface is shown in FIG. 5.The flow field near the platform surface reflects the velocity, vortex and backflow.As can be seen from FIG. 5, vortex and backflow are very easy to occur in the corner of platform and between structures.As can be seen from FIG. 5a, the high pressure area is located at the windward surface. By figure $5 \mathrm{~b}$ and $5 \mathrm{c}$, the fluid in the superstructure of vertical speed when the windward side drops and to tap, at the same time in the superstructure rear back to produce larger vortex as shown in figure $5 \mathrm{c}$, this is because the air viscosity, flow separation phenomenon, caused the surface kona, because of lower velocity caused by the pressure fluid backflow phenomenon.

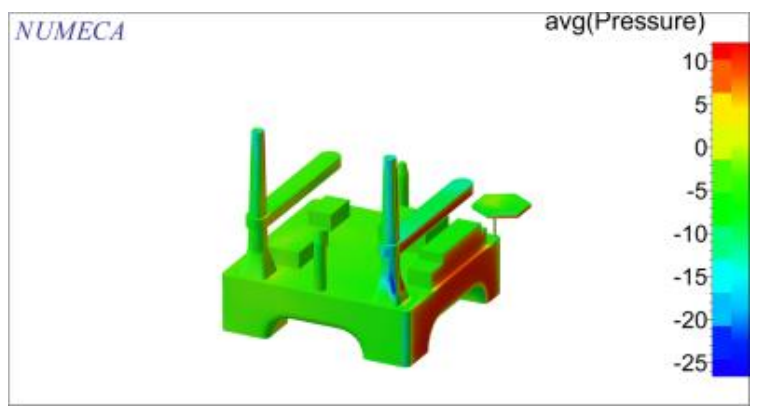

Figure 6 Pressure Nephogram

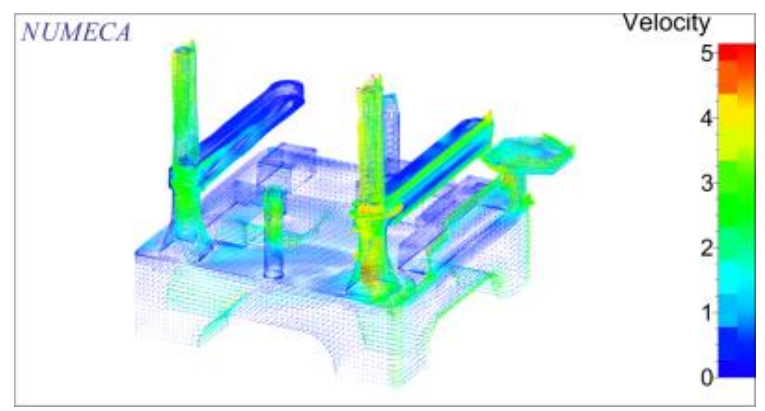

Figure 7 Velocity Vector

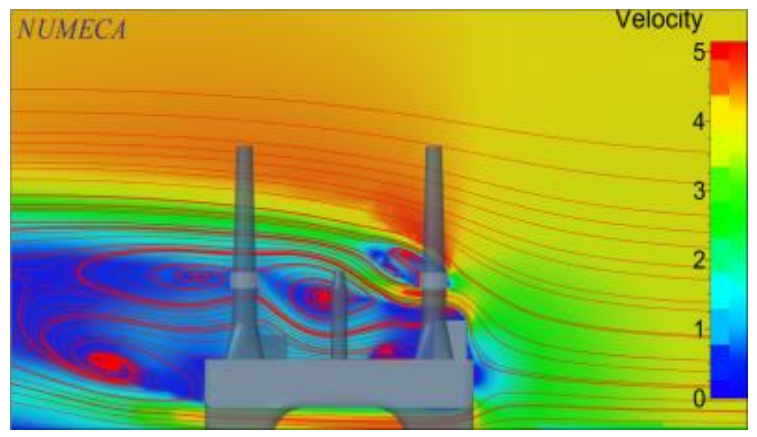

Figure 8 Flow chart

\section{CONCLUSION}

In this paper, an asymmetric semi-submersible lifting platform is taken as the main research object. Based on FINE/MARINE software, the wind loads of platforms with different wind directions are studied, and the following conclusions are drawn:

1) CCS specification calculates the maximum longitudinal force of the platform when wind direction is $165^{\circ}$, the maximum lateral force when wind direction is $60^{\circ}$, the maximum longitudinal force when wind direction is $30^{\circ}$, and the maximum lateral force when wind direction is $315^{\circ}$

2) Due to the asymmetry of the platform, wind loads of $0^{\circ} \sim 180^{\circ}$ and $180^{\circ} \sim 360^{\circ}$ are not the same, but the change trend is basically the same;

3) The maximum resultant force of the platform is calculated by CCS specification when the wind direction is $150^{\circ}$, and the maximum resultant force is calculated by CFD when the wind direction is $30^{\circ}$

4) The standard calculation method cannot fully consider the effect of shading effect among the superstructure components, which results in larger results than the numerical simulation results. When the fluid meets the vertical windward surface of the superstructure, its velocity will plummet and diverge in all directions.

\section{REFERENCES}

[1] Yuan ruhua, wang minglun, li litao, yuan shangchen, Yin hao, wang yufeng. Development status and application of semi-submersible hoisting pipelaying vessel SSCV $[\mathrm{J}]$. China water transport (second half),2017,17(08):1-6+64

[2] Min bing, shu shiyong. Application of semi-submersible lifting engineering ship in ocean engineering $[\mathrm{J}]$. Chinese shipbuilding,2010,51(01):156-161.

[3] Wang zhongchang, yi cong, li da, bai xuoping, zheng wentao, peng chao. Research on FPSO wind load characteristics based on wind tunnel test [J]. Ocean engineering,2016,34(05):125-130.

[4] Furnes G.K. Numerical simulations of wind forces on Troll B[J]. Marine Structures, 11(1998):273-289.

[5] Ma zhe, wang xiao. Numerical simulation of typhoon loads in deep water semi-submersible $[\mathrm{J}]$ science and technology BBS,2009, 08:9-8-99.

[6] Lin yi, hu ankang, xiong fei, et al. Numerical simulation and experimental study of jack-up typhoon loads [J]. Journal of hydrodynamics,2012,27(2):208-215.

[7] Tang jing, wang bo, luo ruifeng. Analysis and research on standard calculation method of jack-up typhoon load [J]. Ship engineering,2016,38(S1):35-38. 